\title{
Huesos de mamíferos (Carnivora: Canidae, Artiodactyla: Cervidae y Lagomorpha: Leporidae) en un depósito cultural de la costa de Jalisco, México
}

\author{
Fabio Germán Cupul-Magaña \\ Joseph B. Mountjoy \\ Centro Universitario de la Costa, Universidad de Guadalajara, Av. Universidad 203, Delegación Ixtapa, C.P. 48280, Puerto \\ Vallarta, Jalisco, México. fabiocupul@ gmail.com
}

Un depósito cultural es el resultado de los procesos de formación del registro arqueológico, definidos como todos los eventos, actividades y procesos que afectan a los artefactos, ecofactos y rasgos después de su uso inicial. Su depositación puede ser por desecho, pérdida accidental, disposición de los muertos y/o el abandono de artefactos aún útiles. A esto le siguen los procesos denominados posdepositacionales, que incluyen diversos agentes naturales o no antrópicos (Schiffer 1991, Renfrew \& Bahn 2007).

En esta nota, documentamos el hallazgo de fragmentos mandibulares de un Perro Canis lupus familiaris Linnaeus, 1758 (Carnivora: Canidae) y un Venado Cola Blanca Odocoileus virginianus (Zimmermann, 1780) (Artiodactyla: Cervidae), así como de fémures y tibia del Conejo Mexicano De Monte Sylvilagus cunicularius (Waterhouse, 1848) (Lagomorpha: Leporidae), recolectados durante el año 2015, dentro del depósito cultural "Arroyo Piedras Azules” en Mexico. Los huesos se identificaron siguiendo a Von den Driesch (1976), Elbroch (2006) y Adams \& Crabtree (2012), así como por comparación directa con material de referencia depositado en la Colección del Laboratorio de Paleozoología del Instituto de Investigaciones Antropológicas y la Colección de la Estación de Biología Chamela del Instituto de Biología de la Universidad Nacional Autónoma de México. Esta última, repositorio de las muestras colectadas y reportadas en este estudio.

"Arroyo Piedras Azules" selocaliza en el municipio de Cabo Corrientes, en el margen oeste del poblado de Maito y distantea 1,5 km de la costa del Pacífico en el estado de Jalisco, México (20.26186 N, -105.57505 O; Fig. 1A). Se ubica en un terreno plano, similar a una península y con una elevación de $29 \mathrm{msnm}$, que se extiende desde las faldas de los cerros al sureste hasta la planicie costera en el noreste. El arroyo Piedras Azules, que da nombre al sitio, se encuentra en la parte Este, y la ocupación prehispánica abarca aproximadamente 3 ha. El terreno parece no haber sido cultivado ni sometido a alguna actividad importante de movimiento de tierra en tiempos históricos. No hay montículos ni otros signos obvios de construcción en la superficie, sino varios fragmentos de cuencas de molienda de metates de granito. También, se ha encontrado cerámica policromada, una estela de granito, figuras antropomorfas de cerámica, objetos de metal, conchas y huesos de diversos grupos taxonómicos.

El sitio "Arroyo Piedras Azules" esta asociado con la cultura Aztatlan del periodo Posclasico Temprano (Mountioy et al. 2016) y fechado por radiocarbono entre el 1165 al 1265 de nuestra era (años calibrados). El fechado por radiocarbono se realizó a partir de dos muestras de carbón vegetal de fogones domésticos, extraídas de las dos capas culturales más profundas del depósito (laboratorio Beta Analytic Inc., La Florida, USA). La más profunda (170-190 cm), descansa sobre la roca madre de granito descompuesto y representa el establecimiento de una colonia del Posclásico Temprano de la cultura arqueológica Aztatlán, mientras que la superior (150-170 cm) corresponde al Posclásico Tardío. El depósito Aztatlán tiene tres o cuatro capas con un grosor de aproximadamente 1 m, en tanto el depósito Posclásico Tardío tiene una o dos capas del mismo grosor. La muestra de carbón vegetal de la capa superior registró un valor en años radiocarbónicos de $810 \pm 30$ AP (Beta-419370), mientras que la muestra de la capa más profunda fue de $800 \pm 30$ AP (Beta-419371).

El 28 de abril de 2015 se recolectó un fragmento mandibular derecho de un ejemplar de Venado Cola Blanca (Fig. 1B) que, por la presencia de los tres molares completamente eruptados y la apariencia aguda de sus cúspides, es probable que corresponda a un animal inmaduro de dos años y medio de edad (McPeake 2012). Este material fue obtenido de un pozo irregular de saqueo (Pozo \#1 de $4 \times 5 \mathrm{~m}$ y ca. $2 \mathrm{~m}$ de profundidad) excavado por un lugareño. El 11 de junio se recolectó, dentro del mismo pozo (80-120 cm), la rama mandibular derecha de un Perro con el canino, tres premolares (p) y un molar (m1). El p3 es deciduo y los p1 y p2 son permanentes que aún están eruptando (Fig. 1C). Este resto, probablemente corresponde a un perro del tipo común de 13 a 15 semanas de edad en proceso de muda dental (R. Valadez com. pers.). El 13 de junio del mismo año se recolectaron tres fémures izquierdos incompletos (Fig. 1D) y uno derecho casi completo, así como una tibia izquierda incompleta (Fig. 1E) de ejemplares adultos de Conejo Mexicano de Monte, dentro del pozo de prueba \#2 (1 $\mathrm{m}^{2}$ y ca. 170-190 cm de profundidad). En ninguno de los huesos se

\footnotetext{
Mammalogy Notes | Notas Mastozoológicas

Sociedad Colombiana de Mastozoología

Vol. 4 Num. 2| 2018
} 


\section{Notas | Notes}

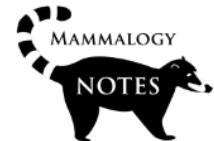

observaron trazas antrópicas o huellas de corte. Por su parte, las especies recolectadas ocurren actualmente en una amplia área geografica que incluye al sitio "Arroyo Piedras Azul es", por lo que no se trata de registros por fuera de sus ambitos distribucionales (Ceballos \& Miranda 2000, Galindo Leal \& Weber 2005, Valadez 2014).

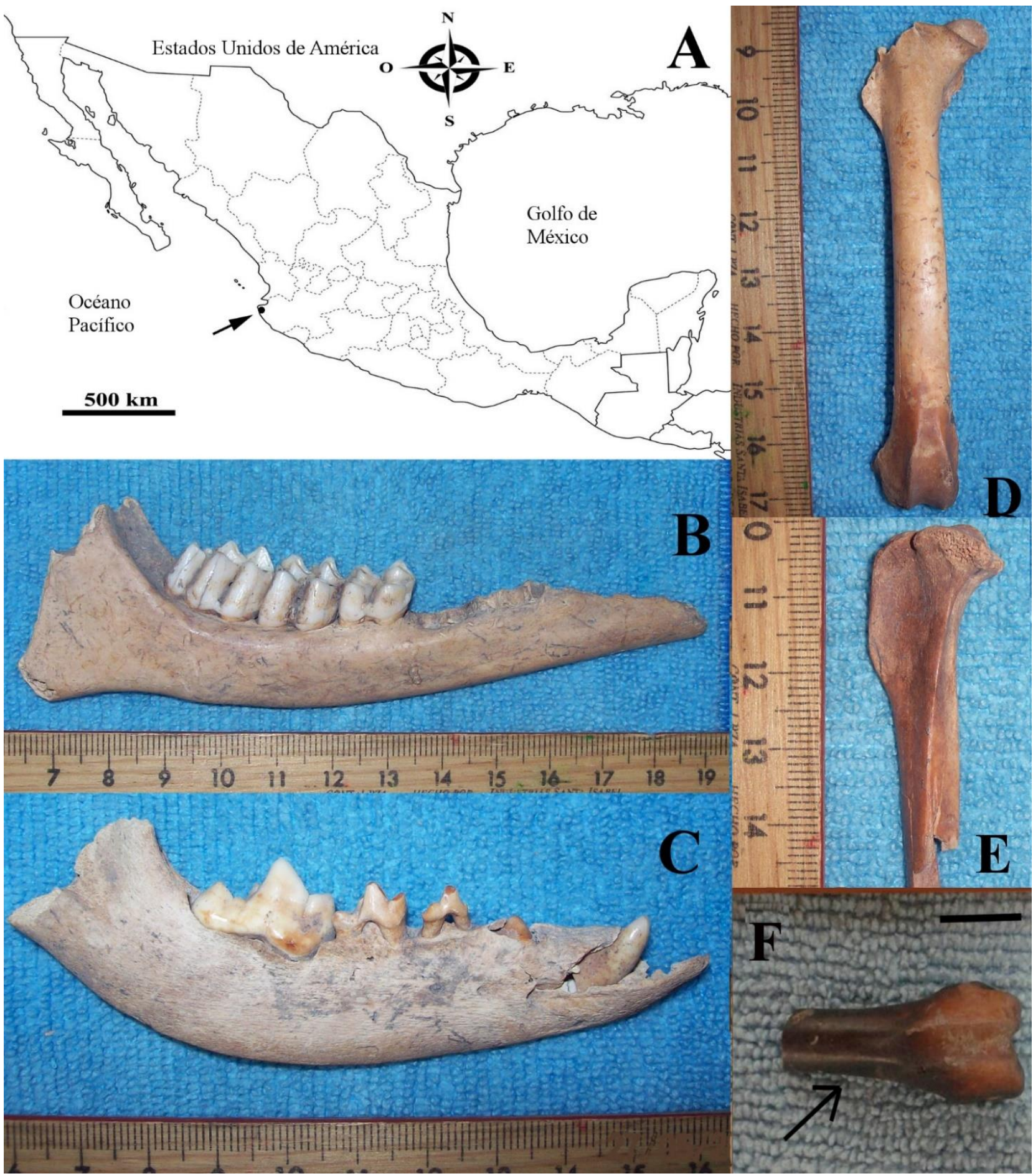

Figura 1. A) Localización de Maito, Jalisco, México (flecha indica su ubicación geográfica). B) Vista lateral de la mandíbula derecha de Odocoileus virginianus. C) Vista lateral de la mandíbula derecha de Canis lupus familiaris. D) Fémur derecho de Sylvilagus cunicularius. E) Tibia izquierda de Sylvilagus cunicularius. F) Parte distal de fémur de Sylvilagus cunicularius del pozo \#4; la flecha señala la porción quemada por contacto secundario con un fuego. Escala de regla de $1 \mathrm{~cm}$ en las figuras 1B, 1C, 1D y 1E. Marca de escala de $1 \mathrm{~cm}$ en la figura 1F.

Mammalogy Notes | Notas Mastozoológicas

Sociedad Colombiana de Mastozoología Vol. 4 Num. 2| 2018 
Estos hallazgos estimularon la implementación de un proyecto de investigación más amplio, cuya primera fase de campo se realizó entre mayo y septiembre de 2017, la cual consistió en la excavación de otros 10 pozos de prospección de 2x2 m. Para la ubicación de los pozos, se tomó en cuenta seguir el perfil del pozo de saqueo, explorar la zona del panteón encontrada por un saqueador, la zona de hallazgo de la estela de granito y la parte plana del sitio de dónde provenía la basura encontrada en la primera excavación. La cala estratigráfica de cada pozo se seccionó en 10 capas, de $10 \mathrm{~cm}$ cada una, desde la superficie hasta $2 \mathrm{~m}$ de profundidad. En los pozos \#1, \#7 y \#10 (capas desde 10 a $90 \mathrm{~cm}$ ), se encontraron fragmentos de dientes y cornamenta de Venado Cola Blanca; en el pozo \#12 (capa de 50 a $60 \mathrm{~cm}$ ), dientes de Perro y en el pozo \#4 (capas desde 140 a $190 \mathrm{~cm}$ ) fragmentos de una tibia, así como de un fémur quemado por posible contacto secundario a una fuente de calor como un fogón (Fig. 1F). Aunque el análisis de estos huesos (y de otros vertebrados y conchas de moluscos) no forma parte de la nota, indican que es posible realizar más descubrimientos conforme se extienden los pozos de prospección por todo el sitio, permitiendo incrementar el número de muestras para un análisis posterior del uso de este tipo de recursos.

Finalmente, la presencia de material quemado (hueso de Conejo Mexicano de Monte) en el sitio “Arroyo Piedras Azules", probablemente evidencie su uso como recurso alimenticio, lo que avalaría la información documentada acerca del aprovechamiento de estas especies como alimento por parte de comunidades prehispánicas en México (Manzanilla \& Serra 1987, Valadez 1993, Mandujano 2004, Gilcrease 2014). Sin embargo, particularmente en el caso del Perro, aunque su utilización como alimento se presenta en los contextos latinoamericanos con alguna frecuencia, posiblemente no era algo muy cotidiano o de uso muy simple, pues se tiene certidumbre de que las personas y los perros compartían alimento en vez de que uno fuera alimento del otro (Valadez 2014). De hecho, en el occidente de México, los hallazgos arqueológicos sugieren que entre los indígenas existía el concepto del Perro como acompañante de los difuntos al más allá (Mountjoy et al. 2014).

\section{Agradecimientos}

A Sylvia Margarita de la Parra y Juan Miguel Ramírez por su apoyo en la revisión de material en la etapa preliminar de este trabajo. A Jill Rhodes por su apoyo en la revisión de material óseo en busca de trazas antrópicas. A Raúl Valadez por su apoyo en la validación de la identificación de los huesos y la consulta de la colección del Laboratorio de Paleozoología. A Enrique Ramírez por su apoyo para consultar la colección de la Estación de Biología Chamela. Al Centro del Instituto Nacional de Antropología e Historia (INAH) en Jalisco, por su permiso la excavar la zona. A Carola Cañón y a los dos revisores anónimos por sus significativos comentarios para mejorar el manuscrito.

\section{Referencias}

ADAMS, B. \& P. CRABTREE. 2012. Comparative osteology: a laboratory and field guide of common North American animals. Academic Press, Oxford, UK 443.

CEBALlOS, G. \& A. MIRANDA. 2000. Guía de campo de los mamíferos de la costa de Jalisco, México / A field guide to the mammals of the Jalisco coast, Mexico. Fundación Ecológica de Cuixmala, A. C. - Universidad Nacional Autónoma de México, México 502.

ELBROCH, M. 2006. Animal skull: a guide to North American species. Stackpole Books, Mechanicsburg, Pennsylvania, USA 727.

GALINDO LEAL, C. \& M. WEBER. 2005. Odocoileus virginianus (Zimmermann, 1789) Venado cola blanca. Pp. 517-521 in Los mamíferos silvestres de México (Ceballos G. \& Oliva G, eds.), Fondo de Cultura Económica - CONABIO, México.

GILCREASE, K. 2014. The Mexican cottontail (Sylvilagus cunicularius): A historical perspective of hunting and grazing and implications for conservation planning. Acta Zoológica Mexicana (n.s.) 30(1):32-40.

MANDUJANO, S. 2004. Análisis bibliográfico de los estudios de venados en México. Acta Zoológica Mexicana (n.s.) 20(1):211-251.

MANZANILLA, L. \& M. C. SERRA. 1987. Aprovechamiento de recursos de origen biológicos en la cuenca de México (2500 antes de Cristo -1500 después de Cristo). Geofísica Internacional 26(1):15-28.

MCPEAKE, R. 2012. Aging Whitetails. University of Arkansas System. Disponible en: https://www.uaex.edu/publications/pdf/FSA-9105.pdf. Fecha de consulta: 16 de mayo de 2016.

MOUNTJOY, J., et al. 2014. El perro en contextos funerarios Valle de Mascota, Jalisco. Arqueología Mexicana 21(125):54-57.

MOUNTJOY, J., et al. 2016. The Early Postclassic Aztatlán colonization of the coast of Jalisco, Mexico. Pp. 309 in Abstracts of the 81st Annual Meeting of Society for American Archaeology (The SSA, eds.), The Society for American Archaeology Press, Orlando, Florida, USA.

RENFREW, C. \& P. BAHN. 2007. Arqueología: teoría, métodos y práctica. Ediciones Akal, S. A., España.

SCHIFFER, M. B. 1991. Los procesos de formación del registro arqueológico. Boletín de Antropología Americana 23:39-45.

VALADEZ, R. 1993. Macrofósiles faunísticos. Pp. 729-831 in Anatomía de un conjunto residencial teotihuacano en Oztoyahualco. II Estudios Específicos. (L. Manzanilla, ed.), Instituto de Investigaciones Antropológicas - UNAM, México.

VALADEZ, R. 2014. El origen del perro americano y su dispersión. Arqueología Mexicana 21(125):30-37.

VON DEN DRIESCH, A. 1976. A guide to the measurement of animal bones from archaeological sites. Peabody Museum Bulletin 1. Harvard University, Cambridge, Massachusetts, USA 136. 